\title{
REZENSIONEN
}

\section{Frank Deckers „Architektur der deutschen Politik“: fulminanter Entwurf mit einigen Baulücken. Ein Rezensionsessay}

\author{
Eckhard Jesse
}

\section{Decker, Frank: Regieren im „Parteienbundesstaat“. Zur Architektur der deutschen Politik, VS Verlag für Sozialwissenschaften, Wiesbaden 2011, 347 Seiten, € 29,95.}

Frank Decker ist einer der über die Fachgrenzen hinaus bekannten Politikwissenschaftler Deutschlands, nicht zuletzt bedingt durch seine vielfältige mediale Präsenz - im Fernsehen, im Radio, in Tages- und Wochenzeitungen, ebenso in nicht auf die scientific community ausgerichteten Organen wie „MUT“ und „Berliner Republik“. Er sitzt keineswegs im „Elfenbeinturm“, bezieht oft gesellschaftspolitisch Stellung und packt „heiße Eisen“ an. Das gilt zumal für sein wissenschaftliches Engagement. Decker, der den - nicht persönlichen Konflikt unter Kollegen geradezu sucht, hat mancher wissenschaftlichen Position den Fehdehandschuh hingeworfen - so der von Katharina Holzinger und Christoph Knill, die vor zehn Jahren Joschka Fischers Appell für eine präsidentielle Demokratisierung der EU nichts abgewinnen konnten ${ }^{1}$, so Gerd Strohmeiers Plädoyer für ein mehrheitsbildendes Wahlsystem $^{2}$, so zuletzt Steffen Kailitz' Auffächerung der Parlamentarismus-Präsidentialismus-Dichotomie. ${ }^{3}$ In fast allen Fällen ging die Initiative zum Widerspruch von Decker aus. ${ }^{4}$ Dieser konfliktorientierte Argumentationsstil steht in einem gewissen Gegensatz zu seiner mehr oder weniger starken Präferenz für konkordanzdemokratische Elemente.

Deckers Studien zur „Architektur der deutschen Politik“ stellen eine „vorläufige Quersumme“ (S. 9) seiner Beschäftigung mit dem deutschen Regierungssystem dar. Der „rote Faden“ ist die Frage, „wie das Zusammenwirken von mehrheits- und konsensdemokrati-

1 Vgl. Katharina Holzinger / Christoph Knill, Institutionelle Entwicklungspfade im Europäischen Integrationsprozess: Eine konstruktive Kritik an Joschka Fischers Reformvorschlägen, in: ZPol, 11. Jg. (2001), H. 3, S. 987 - 1010; Frank Decker, Institutionelle Entwicklungspfade im europäischen Integrationsprozess. Eine Antwort auf Katharina Holzinger und Christoph Knill, in: ZPol, 12. Jg. (2002), S. $611-636$.

2 Vgl. Gerd Strohmeier, Ein Plädoyer für die „gemäßigte Mehrheitswahl“: Optimale Lösung für Deutschland, Vorbild für Österreich und andere Demokratien, in: ZParl, 38. Jg. (2007), H. 3, S. 578 - 590; Frank Decker, Konsens- oder mehrheitsdemokratischer Wandel im Parlamentarismus? Eine Replik auf Gerd Strohmeier in Heft 3/2007 der ZParl, in: ZParl, 38. Jg. (2007), H. 4, S. 841 - 845; Gerd Strohmeier, Die (gemäßigte) Mehrheitswahl im Streit der Wissenschaft. Eine Antwort auf Frank Decker, Harald Schoen und Stefan Köppl, in: ZParl, 39. Jg. (2008), H. 2, S. 419 - 425.

3 Vgl. Steffen Kailitz, Ein Unterschied wie Tag und Nacht? Fraktionsgeschlossenheit im Parlamentarismus und Präsidentialismus, in: ZPol, 18. Jg. (2008), H. 2, S. 291 - 324; Frank Decker, Ist die Parlamentarismus-Präsidentialismus-Dichotomie überholt? Zugleich eine Replik auf Steffen Kailitz, in: ZPol, 19. Jg. (2009), H. 1, S. 169 - 203; Steffen Kailitz, Zur Typologisierung der Regierungsformen. Eine Antwort auf Frank Deckers Replik, in: ZPol, 20. Jg. (2010), H. 1, S. 47 - 77.

4 Siehe allerdings: Frank Decker, Zwischen Placebo und Erfolgsmodell. Direkte Demokratie auf der Landesebene, in: ZParl, 41. Jg. (2010), H. 3, S. 564 - 579; Everhard Holtmann, Direkt gewählte Ministerpräsidenten der Länder - eine kritische Folgenabschätzung der von Frank Decker in Heft 3/2010 der ZParl veröffentlichten Überlegungen, in: ZParl, 42. Jg. (2011), H. 1, S. $194-205$. 
schen Merkmalen den Regierungsprozess in der Bundesrepublik beeinflusst" (S. 9). Der erste, zentrale Beitrag analysiert den deutschen „Parteienbundesstaat“ im Spannungsverhältnis von Mehrheits- und Konsensdemokratie - das Leitthema des Bandes. Die Typologien von Arendt Lijphart, der dieser dichotomischen Unterscheidung folgt, und Roland Czada, bei dem eine Vielfalt an Kombinationen zur Geltung kommt, sind für ihn der Fixpunkt, um das „deutsche Modell“ einzuordnen. Decker arbeitet drei spezifische Merkmale heraus: Erstens überlagerten die Elemente des Verfassungsstaates die der Demokratie, wirkten also eher konsensdemokratisch. Diese teils weithin akzeptierten, teils kritisierten Vetostrukturen rechtfertigten die Einordnung als „semi-souveräner Staat“ (Peter J. Katzenstein). Hingegen sei das gewaltenfusionierende parlamentarische System - zweitens - stark durch mehrheitsdemokratische Merkmale geprägt. Das eine „Lager" regiert, das andere opponiert. Allerdings werde die Mehrheitsbildung zunehmend schwieriger. Drittens schließlich ist durch die korporatische Interesseneinbindung, etwa durch die funktionierende Tarifpartnerschaft zwischen Arbeitgebern und Arbeitnehmern, viel Konsens erreicht worden, ungeachtet zahlreicher Erosionstendenzen in der letzten Zeit.

Leider berücksichtigt Decker die verschlungene Geschichte des Begriffs des „Parteienbundesstaates", der immerhin den Titel seiner Studie ziert, nicht näher. Dieser vom Staatsrechtslehrer Carl Bilfinger Ende der Weimarer Republik geprägte Terminus ${ }^{5}$ hatte seinerzeit eine pejorative Konnotation - Parteien instrumentalisierten das föderalistische Gefüge. Der Parteienstaat überlagere den Bundesstaat. ${ }^{6}$ Als in den 1970er Jahren die unterschiedlichen Mehrheiten im Bundestag und im Bundesrat zu heftigen Konflikten zwischen der SPD und der FDP auf der einen, der Union auf der anderen Seite führten ${ }^{7}$, griffen SPD-Politiker diese Wendung auf, wandten sich gegen die parteipolitische Inanspruchnahme des Bundesrates $^{8}$ - unter anderem unter Berufung auf Theodor Maunz, der davor gewarnt hatte, „dass aus dem Bundesstaat ein ,Parteien-Bundesstaat' wird“" . Der konservative Staatsrechtslehrer Maunz votierte gegen jede „parteipolitische Konfrontation“ bei der Arbeit des Bundesrates: „Das Grundgesetz will nicht, wenn man einen Begriff der Weimarer Verfassung verwenden will, einen ,Parteienbundesstaat` schaffen, das heißt, hier ein bundesstaatliches System aufbauen, in dem die politischen Positionen dazu verwendet werden, um aus parteipolitischer Haltung heraus vom Lande her gegen die Bundesregierung Opposition zu machen. " ${ }^{10}$ Die

5 Vgl. Carl Bilfinger, Exekution, Diktatur und Föderalismus, in: Deutsche Juristenzeitung, 37. Jg. (1932), Sp. 1017 - 1021. Der Autor hatte hier den „Preußen-Schlag“ von Papens gerechtfertigt. Carl Schmitt griff diesen Terminus im gleichen Jahr in zwei Aufsätzen auf. Vgl. ders., Konstruktive Verfassungsprobleme (1932) sowie Starker Staat und gesunde Wirtschaft (1932), in: ders., Staat, Großraum, Nomos. Arbeiten aus den Jahren 1916-1969, herausgegeben von Günter Maschke, Berlin 1995, S. 55 - 70, S. 60 sowie S. 71 - 91, S. 72.

6 Vgl. Ernst Rudolf Huber, Deutsche Verfassungsgeschichte seit 1789, Bd. VI: Die Weimarer Reichsverfassung, Stuttgart u.a. 1981, S. 59 f., S. 375 f.

7 Vgl. Friedrich Karl Fromme, Gesetzgebung im Widerstreit. Wer beherrscht den Bundesrat? Die Kontroverse seit 1969, Bonn 1980.

8 Vgl. Gerhard Jahn, Tendenzen zum „Parteienbundesstaat“ seit 1969, in: ZParl, 7. Jg. (1976), H. 3, S. $291-297$.

9 Zitiert nach ebenda, S. 293.

10 So Theodor Maunz, Die Rechtsstellung der Mandatsträger im Bundesrat, in: Bundesrat (Hrsg.), Der Bundesrat als Verfassungsorgan und politische Kraft. Beiträge zum fünfundzwanzigjährigen Bestehen des Bundesrates der Bundesrepublik Deutschland, Bad Honnef / Darmstadt 1974, S. $193-211$. 
Paradoxie besteht darin, dass die seinerzeit im Bund regierende SPD sich damit eine - konservative - Position zueigen machte, die das parteienstaatliche Element zugunsten des bundesstaatlichen zurückzudrängen suchte. In der Politikwissenschaft findet der Begriff des „Parteienbundesstaates" heutzutage kaum Verwendung. Wenn das dennoch geschieht, ist die einst abwertende Konnotation verloren gegangen. Ein Beispiel dafür bietet Klaus von Beyme. Von der ersten Auflage seines „Politischen Systems der Bundesrepublik Deutschland“ an gibt es ein Unterkapitel „Der ,Parteienbundesstaat““. ${ }^{11}$ Dieser gilt nun keineswegs als Übel - ungeachtet der Blockademacht des Bundesrates. Im Gegenteil: Das Wort von der „Bundestreue“, auf das die Gegner des „Parteienbundesstaates“ sich stützen, firmiert zum Teil als vordemokratisches Relikt, das sich wenig dazu eigne, „Verhaltensregeln für die Länder abzuleiten"12.

Reformperspektiven, diese Blockaden zu reduzieren, sieht Decker entweder in der Schwächung der Rolle des Bundesrates oder in der „Mäßigung“ des Parteienwettbewerbs. Beide Wege hätten zu wenig Erfolg geführt. So schlägt er vor, die blockierte Demokratie durch plebiszitäre Elemente zu unterlaufen. Die regierende Mehrheit würde dadurch Minderheiteninteressen berücksichtigen, und der stärkere Einfluss des Volkes sorgte für mehr Konsens (und garantierte Entscheidungsfähigkeit). Was Decker präsentiert, ist originell aber ist es auch praktizierbar?

Deckers Werk, weder ein herkömmliches Lehrbuch zum politischen System der Bundesrepublik Deutschland noch eine disparate Aneinanderreihung bereits publizierter Abhandlungen, siedelt in der Tat ,in der Mitte zwischen einer ,bloßen“ Aufsatzsammlung und einer geschlossenen monographischen Abhandlung“ (S. 9). Trotz der zum großen Teil an anderer Stelle veröffentlichten und nun erweiterten Beiträge (die Zahl der „Nachweise“ auf S. 397 wäre zu ergänzen) bleiben Überschneidungen und Wiederholungen begrenzt; allerdings konnte ein Band aus einem Guss angesichts dieser „Vorgeschichte" nicht entstehen. Nach dem Einleitungsbeitrag zum „Parteienbundesstaat“ folgt ein Aufsatz zu den Leistungen und Defiziten des Parlamentarismus. Das dritte Kapitel enthält drei Beiträge - zu den Grundlagen und Problemen der Parteiendemokratie, den Koalitionsoptionen und -aussagen im Fünfparteiensystem (mit Volker Best) sowie zur Frage nach der Änderung des Wahlsystems, das vierte besteht aus einem umfassenden Text (dem längsten) zur Diskussion um die Einführung plebiszitärer Elemente, im fünften geht es in drei Abhandlungen um die neue Abweichungsgesetzgebung, die notwendige Reform des Bundesrates und um die Struktur der Regierungssysteme in den Ländern. Das Sammelwerk schließt mit einem Beitrag zum Präsidentenamt in der Parteiendemokratie.

Der Autor erwähnt zahlreiche Missverständnisse in der Öffentlichkeit (und zum Teil in der Rechtswissenschaft) über die Funktionsweise des parlamentarischen Systems, so zur Gewaltenteilungsidee (als stehe das gesamte Parlament der Regierung gegenüber), zur „funktionellen“ Gewaltenteilung (als obliege dem Parlament die Gesetzgebung und der Regierung die Ausführung der Gesetze) sowie zur Art und Weise der Entscheidungsfindung (als solle im Plenum um die „besten Lösungen“ gerungen werden, unabhängig von der politischen Konstellation). Das Denken in den traditionellen Bahnen des Konstitutionalismus gefährde beziehungsweise erschwere nach wie vor das Verständnis für die Mecha-

11 Vgl. Klaus von Beyme, Das politische System der Bundesrepublik Deutschland, Wiesbaden 2010, S. $373-376$.

12 Ebenda, S. 373. 
nismen der parlamentarischen Demokratie in Deutschland. Deren Leistungen sieht Decker in der Regierungsstabilität und im funktionierenden Parteiensystem, das ein hohes Maß an Fraktionsdisziplin gewährleiste. Der „Zwang zur Geschlossenheit“ (S. 71) um jeden Preis unterminiere jedoch die innerparteiliche Demokratie und schränke den Handlungsspielraum der Parlamentarier ein. Insgesamt sei der legislative Einfluss der Parlamentsmehrheit nirgendwo in Europa so gering wie in der Bundesrepublik. Ob das stimmt, ist zu bezweifeln. Wenn es stimmt, wären dann nicht stärkere Konkurrenzmechanismen angebracht?

Was den Komplex des Parteiensystems und der Regierungsbildung betrifft, erörtert Decker aktuelle Problemlagen, so bei der berechtigten Kritik ${ }^{13}$ an der „Selbstprivilegierung der Parteien im Parteienstaat" (mit Blick auf die indirekte Staatsfinanzierung der Parteien, das Rekrutierungsmonopol des politischen Personals und die Ämterpatronage). Mit „Koalitionsoptionen und -aussagen" greift der Autor ein bisher weithin unbeachtetes Themenfeld auf - im Gegensatz etwa zu der zum Teil etwas blutleeren szientistischen Koalitionsforschung. Die Parteien hätten vor der Bundestagswahl 2009 durch „Ausschließeritis“ (Tarek Al-Wazir) jegliche Dreierkoalition blockiert. ${ }^{14}$ Für die FDP schied eine Koalition mit der SPD aus, allenfalls erwog sie eine solche mit der CDU und den Grünen. Diese hingegen schlossen ein Bündnis mit den Liberalen und der CDU eigens aus, aber nicht mit der Linken. Die SPD konnte sich im Notfall eine Ampelkoalition mit den Grünen und den Liberalen vorstellen, jedoch keine mit der Linken. Ohne eine schwarz-gelbe Mehrheit wäre damit nur ein - erneutes - schwarz-rotes Bündnis in Frage gekommen. Allerdings ist der Schluss, den Decker aus der komplizierter gewordenen Koalitionsbildung zieht, demokratietheoretisch höchst fragwürdig: „Da die Ampel-Koalition für die FDP [...] fürs erste keine Rolle mehr spielen wird, bleibt der SPD nur die Möglichkeit, sich die Machtoption Rot-Rot-Grün zu erschließen, wenn sie sich nicht mit der Rolle eines Juniorpartners in Großen Koalitionen begnügen will." (S. 126) Wo bleibt da der antiextremistische Konsens? Machtpolitik darf bei der Koalitionsbildung nicht der einzige Aspekt sein, was ein anspruchsvoller „Reformdemokrat“ wie Decker weiß. Wenn der Autor an anderer Stelle kühn meint, es konnte und kann „bei allen berechtigten Vorbehalten gegenüber den Postkommunisten“ (S. 323) nicht die Rede davon sein, dass sie [die Linke] „zum nicht-demokratischen Teil des politischen Spektrums gerechnet werden müsse“ (S. 322 f.), so fallen - nicht nur an dieser Stelle - vielfältige Erkenntnisse der normativen Extremismusforschung, für die die Linke wahrlich kein Gralshüter des demokratischen Verfassungsstaates ist, auch wenn sie sich vom harten Extremismus der NPD abhebt ${ }^{15}$, unter den Tisch. Warum sollte ungeachtet momentan divergierender Positionen in absehbarer Zeit nicht wieder ein Bündnis der FDP mit der SPD in Frage kommen? Die Sozialdemokraten haben im Bundestagswahlkampf 2009 den Liberalen als Koalitionspartner den Vorrang gegenüber der Linken gegeben. Deckers Argumentation nimmt in diesem Punkt die „lebende Verfassung“ (Dolf

13 Sie fällt im Vergleich zu der Kritik von Hans Herbert von Arnim differenzierter aus. Vgl. ders., Das System. Die Machenschaften der Macht, München 2001; ders., Volksparteien ohne Volk. Das Versagen der Politik, München 2009.

14 Das heißt im Umkehrschluss aber nicht, dass jede Koalition prinzipiell in Frage kommen muss.

15 Vgl. Eckhard Jesse, Die NPD und die Linke. Ein Vergleich zwischen einer harten und einer weichen Form des Extremismus, in: Uwe Backes / Alexander Gallus / Eckhard Jesse (Hrsg.), Jahrbuch Extremismus \& Demokratie, Bd. 21, Baden-Baden 2009, S. 13 - 31. 
Sternberger) unzureichend zur Kenntnis, verabsolutiert Momentaufnahmen - als seien Mehrheiten und Koalitionen festgezurrt.

Bei der Frage nach der Koalitionsbildung laviert der Autor. Wer einerseits am Konkurrenzgedanken festhalten will (also kein lagerübergreifendes Dreierbündnis favorisiert) und andererseits die Linke wegen ihrer demokratietheoretisch problematischen Position nicht in das Regierungsgefüge eingliedern möchte, kann einen Ausweg über eine (in Deutschland aufgrund der Last der Vergangenheit eher verpönte) Minderheitsregierung finden. Wenn die schwarz-gelbe Konstellation besser abschneidet als die rot-grüne, aber ohne Mehrheit bleibt, so müssten die Grünen bereit sein, eine schwarz-gelbe Koalition zu tolerieren; im umgekehrten Fall sollte das für die Liberalen ebenso gelten. Auf diese Weise sind die beiden großen Parteien in der Regierung und in der Opposition. Gewiss ist es für die FDP und die Grünen nicht ganz einfach, ein solches Verhalten ihren Wählern gegenüber einleuchtend zu begründen, doch würde dieses Glaubwürdigkeit ausstrahlen. Im Übrigen zeigt sich an den Wahlen 2011 in Rheinland-Pfalz, Baden-Württemberg und Bremen, dass es unverändert für „kleine“ Koalitionen reicht, in Hamburg sogar für eine Alleinregierung. Als Nicht-Gegner von Minderheitsregierungen sollte Decker einer solchen Konstellation nicht negativ gegenüberstehen.

Die Frage, ob ein neues Wahlsystem notwendig sei, beantwortet der Autor überzeugend mit einem klaren Nein, was den Systemwechsel von der Verhältniswahl zur Mehrheitswahl betrifft ${ }^{16}$ - nicht zuletzt deshalb, weil eine angemessene Legitimation für die faktische Ausschaltung kleiner Parteien aus dem Politikbetrieb fehlt. Hingegen plädiert er zu Recht nicht nur, wie vom Bundesverfassungsgericht angeordnet, für eine Beseitigung des ,inversen Erfolgswerts", sondern auch für eine Abschaffung der Überhangmandate, um jeglicher Manipulation entgegenzutreten. Decker kann dem Vorschlag des Rezensenten, im Bund zum Einstimmensystem (von 1949) zurückzukehren, aus Gründen der Transparenz Positives abgewinnen, kritisiert jedoch folgenden Punkt: „Jesse [...] erhofft sich von der Rückkehr zum Einstimmensystem außerdem eine Aufwertung der Personenwahl. Dies erscheint jedoch arg spekulativ, da sich die Wähler dann vermutlich erst recht an der Parteizugehörigkeit der Kandidaten orientieren würden." (S. 160) Das dürfte stimmen. Nur: Gegenwärtig spielt bei einem direkt gewählten Abgeordneten die Höhe der Differenz zwischen der Erst- und der Zweitstimme keinerlei Rolle. Gewiss mag bei einem Einstimmensystem der Wähler in erster Linie „seiner“ Partei folgen. Doch wenn bloß in einem Prozent der Fälle die Wahlbürger wegen der Persönlichkeit des Kandidaten diesem die Stimme geben (und nicht dem Bewerber „ihrer" Partei), hat dies eine effektive Konsequenz, keine bloß symbolische. Insofern wertet das Einstimmensystem, das weitere Vorteile aufweist ${ }^{17}$, die personelle Komponente auf.

Der lange Aufsatz über „Das Volk als Gesetzgeber“, ein Kleinod, verbindet in gewisser Weise die drei vorhergehenden Beiträge zum Parteienwettbewerb mit den drei nachfolgenden zum Föderalismus. Deckers Position „zwischen den Fronten“ wirft der „plebiszitären

16 Allerdings ist die Einordnung der Wahlsysteme nicht durchweg triftig. Eine „Verhältniswahl in Mehrpersonenwahlkreisen" muss nach dem Repräsentationsprinzip nicht Verhältniswahl, sondern kann auch Mehrheitswahl sein (und zwar dann, wenn im Mehrpersonenwahlkreis nur wenige Mandate zu gewinnen sind).

17 Vgl. Eckhard Jesse, Wahlrecht zwischen Kontinuität und Reform. Eine Analyse der Wahlsystemdiskussion und der Wahlrechtsänderungen in der Bundesrepublik Deutschland 1949-1983, Düsseldorf 1985, S. $307-311$. 
Richtung" mit der Fixierung auf die Volksgesetzgebung einen falschen Ansatz vor, ohne dass er deswegen das plebiszitäre Element gänzlich ad acta legt. Die Ausgestaltung der direkten Demokratie ist in den Ländern fortgeschritten. Jedoch kommen durch hohe Quoren und weitreichende Ausschlussgegenstände Plebiszite nicht recht zur Geltung. Der Autor schlägt für den Bund daher den Verzicht auf die Gesetzesinitiative durch das Volk vor. Stattdessen basiert sein - in einem komplexen Argumentationsgang entfaltetes - systemverträgliches Instrumentarium der direkten Demokratie auf vier austarierten Elementen: einer Gesetzesinitiative (ihr komme lediglich eine konsultative Funktion zu), einer Vetoinitiative (das Volk erhält die Möglichkeit, ein bereits beschlossenes Gesetz zu Fall zu bringen), im Gegenzug einem (einfachen) Referendum (die Regierung kann Gesetzesbeschlüsse des Bundestages dem Volk vorlegen, um sie gegen ein ablehnendes Votum des Bundesrates auf diese Weise in Kraft zu setzen) und einem obligatorischen Referendum (etwa bei wichtigen internationalen Verträgen oder bei grundlegenden Verfassungsrevisionen). Gemäß Decker würde durch ein solches „Design“ der direkten Demokratie ein stärker konsensorientierter Politikstil Einzug halten, der zugleich die Blockade des „Parteienbundesstaates“ minderte. Aber ist das, was gewünscht wird, angesichts populistischer Versuchungen auch zu erwarten?

Für den Autor gilt die Föderalismusreform der letzten Großen Koalition, die stärker der Entflechtung zwischen Bund und Ländern Rechnung tragen sollte, weithin als missglückt. Die Zuordnung der Verantwortlichkeiten sei nicht klarer geworden, der Anteil der nicht zustimmungsbedürftigen Gesetze weniger stark gestiegen als erhofft. Bei den konfliktträchtigen Politikfeldern hat sich in der Tat kaum etwas geändert. Eine Reform der Abstimmungsregeln im Bundesrat sieht Decker als nötig an. Da sie für dessen Beschlüsse eine absolute Mehrheit vorschreiben, zählen Enthaltungen faktisch als Nein-Stimmen. Das erschwert bei Zustimmungsgesetzen das Zustandekommen des Gesetzes, bei Einspruchsgesetzen erleichtert es dies. Weil sich die Koalitionsfärbung in den Ländern nicht überall mit der Konstellation im Bund deckt, enthält sich das jeweilige Land bei einer „gemischten“ Zusammensetzung (im Fall einer Meinungsverschiedenheit). Dadurch (und durch das Wählervotum, das bei den „Zwischenwahlen“ die im Bund regierenden Parteien überwiegend nicht begünstigt) sind die Regierungsparteien im Bundesrat oft ohne Mehrheit. ${ }^{18}$ Das erschwert das „Durchregieren“. Der Autor plädiert für die Umkehrung der Abstimmungsfrage: Nicht nach der Zustimmung zum Gesetz sei zu fragen, sondern nach seiner Verweigerung. Auf diese Weise zählten Enthaltungen faktisch als Ja-Stimmen. So würde bei zustimmungsbedürftigen Gesetzen der Einfluss des Bundesrates geschwächt - und damit der „Parteienbundesstaat“. Doch die Schieflage bliebe unverändert. Enthaltungen sind nun einmal Enthaltungen. Die beste Lösung (nicht für Decker): Enthaltungen fallen unter den Tisch, die relative Mehrheit gilt. Dass die demokratischen Parteien einen solchen (vielfach erörterten) Vorschlag nicht umsetzen, ist ein symptomatisches Zeichen für Reformstau im "Parteienbundesstaat".

Deckers Analyse der Regierungssysteme in den Ländern arbeitet mit Blick auf die Volksgesetzgebung viele und mit Blick auf das Wahlsystem wenige Unterschiede zum Bund heraus. In den Ländern hat sich wie im Bund die parlamentarische Regierungsform etabliert - wenn auch mit Variationen in den Modalitäten: So besitzen alle Landesparlamente das Recht der Selbstauflösung mit absoluter oder mit Zweidrittelmehrheit. Manche Autoren

18 Dies traf selbst für die Große Koalition nach der hessischen Landtagswahl im Januar 2009 zu (mit der Bildung einer schwarz-gelben Koalition). 
halten die parlamentarische Regierungsform in den Ländern bekanntlich keineswegs für adäquat - nicht zuletzt wegen der Auszehrung der Gestaltungskompetenzen. Decker bringt dieser Position beträchtliche Sympathien entgegen. Der Exekutivföderalismus überlagere die Rolle der Landtage. Als mögliche Varianten sieht der Bonner Politikwissenschaftler das Konkordanzmodell schweizerischer Prägung, das Wilhelm Hennis bereits 1956 vorgeschlagen hatte und das auf eine Allparteienregierung hinausläuft, oder das präsidentielle Modell, wie es Theodor Eschenburg 1952 für den neuen Südweststaat in Erwägung gezogen hatte. Decker votiert für ein präsidentiell-parlamentarisches Mischsystem, bei dem der vom Volk gewählte Ministerpräsident zugleich vom Parlament abhängig bleibt. Volksgesetzgebung sei mit einem solchen Regierungstypus besser vereinbar als mit dem parlamentarischen System. Gewiss: In den Kommunen ist mittlerweile eine Präsidentialisierung erfolgt. Nur: Ist dies auf die Landesebene übertragbar? Hier wäre ein Blick über die Grenzen - wie an anderen Stellen geschehen - hilfreich gewesen, zum Beispiel nach Israel, das die Direktwahl des Ministerpräsidenten bei gleichzeitiger Abhängigkeit vom Parlament nach kurzer Praxis wieder abgeschafft hat.

Hingegen plädiert Frank Decker beim Amt des Bundespräsidenten nicht für eine Direktwahl (sie sei kaum mit dem parlamentarischen Regierungssystem kompatibel, da ein Wahlkampf Erwartungen weckte, die angesichts der bescheidenen Rolle des Präsidenten unerfüllt bleiben müssten), sondern für eine stärker konsensuelle Lösung. Diese wäre durch ein höheres Quorum bei der Bestellung zu erreichen (zum Beispiel Zweidrittelmehrheit im ersten und zweiten Wahlgang; absolute Mehrheit im dritten Wahlgang - was aber ist, wenn eine solche Mehrheit auch im dritten Wahlgang nicht zustande kommt? Zum nicht-parteilichen Amtsverständnis des Präsidenten passe ein solches Verfahren besser als das bisherige. Obwohl (nicht nur) dieser Beitrag immer wieder aktuelle Beispiele heranzieht, klammert Decker das jüngste - Joachim Gauck versus Christian Wulff nach dem Rücktritt von Horst Köhler - leider aus und verweist stattdessen auf seinen andernorts publizierten Aufsatz. ${ }^{19}$ Dabei hätte gerade diese Wahl reichhaltiges Anschauungsmaterial für Deckers Grundthese von der Notwendigkeit eines größeren Konsensus geliefert.

Die Argumentation zielt auf zentrale Felder der deutschen Politik: Parteien(-system), Föderalismus, Koalitionspolitik, plebiszitäre Elemente, Wahlrechtsgesetzgebung. Der Autor ist mithin ein „Generalist“, den Gefechte auf „Nebenkriegsschauplätzen“, die vom Kern seines Anliegens wegführen, nicht interessieren. Exzentrische Randpositionen sind ihm ohnehin nicht eigen. Eine spezifische Stärke des Werkes besteht zum einen im Bestreben eine Fülle aktueller Beispiele für die eigenen Thesen heranzuziehen, sei es im bestätigenden, sei es im widerlegenden Sinn; zum anderen in zahlreichen Verweisen zum Ausland, die sinnvollerweise mehr der Illustration dienen, jedenfalls keine Systematik anstreben. Insofern ist der Vergleich asymmetrisch. ${ }^{20}$ Wer das deutsche Regierungssystem in seinen Verästelungen durchdringt, muss andere politische Systeme kennen. Vor allem sind Deckers - systemverträgliche - Reformperspektiven erhellend: So bedürfe es der Prüfung, wie sich die Revision einer Systemeigenschaft unter Umständen auf eine andere auswirkt. Historische Prägungen und politische Interessenlagen seien stets zu berücksichtigen. „Es reicht eben nicht, sich auf

19 Vgl. Frank Decker, Das Präsidentenamt in der Parteiendemokratie. Eine Nachlese zum Grundsätzlichen aus politikwissenschaftlicher Sicht, in: MUT, H. 9/2010, S. 36 - 43.

20 Hier ähnelt der Band der Studie von Ludger Helms, Die Institutionalisierung der liberalen Demokratie. Deutschland im internationalen Vergleich, Frankfurt am Main 2007. 
der grünen Wiese Idealmodelle auszumalen und dann an die Politik zu appellieren, sie möge diese doch bitte verwirklichen. Analysen, die so argumentieren, bleiben ganz im Voluntaristischen befangen und können allenfalls den heuristischen Wert einer Utopie für sich reklamieren." (S. 272) Dem ist nichts hinzuzufügen.

Obwohl die Beiträge zum Teil zu unterschiedlichen Zeiten entstanden sind, finden sich kaum Ungereimtheiten und Inkonsistenzen, wenngleich Deckers „Parteienbundesstaat“ keine ganz klaren Konturen hat. Nach welcher Seite hin ist das Spannungsverhältnis zwischen Parteien- und Bundesstaat aufzulösen? Die Frage lautet, ob die plebiszitären Elemente tatsächlich die erhofften Wirkungen entfalten. Seine Position zu den Koalitionsaussagen der Parteien vor Wahlen ist in einem wesentlichen Punkt unstimmig. Einerseits heißt es bei ihm, die Wähler müssten akzeptieren, dass „nicht sie, sondern Parteien beziehungsweise Parteiführungen über die Regierungsbildung entscheiden“ (S. 102; ähnlich S. 117), andererseits: Koalitionsaussagen seien „aus demokratischer Sicht begrüßenswert, denn sie erhöhen den in Vielparteiensystemen reduzierten Einfluss des Wählers auf die Regierungsbildung“ (S. 109). Solche Festlegungen gelten als ein „probates Mittel, die jeweils besten Elemente der Mehrheits- und Konsensdemokratie miteinander zu verbinden“ (S. 109).

In gewisser Weise erinnern die Beiträge Frank Deckers an jene von Theodor Eschenburg, einen der Gründungsväter der deutschen Politikwissenschaft. Wie kaum ein anderer wusste Eschenburg, sechs Jahrzehnte vor Decker geboren (1904), seine wissenschaftlichen Erkenntnisse als eine Art „praeceptor Germaniae“ in die Öffentlichkeit zu tragen. ${ }^{21}$ Mit Eschenburg hat Decker die verständliche und klare Sprache gemein, ferner die pointierte Sichtweise und das Gespür für Praxisrelevanz. Ist es nur ein Zufall, dass kein zweiter deutscher Wissenschaftler im Personenregister so häufig auftaucht wie der 1999 gestorbene Tübinger Gelehrte? Der Bonner besitzt aber weder auctoritas noch potestas von Eschenburg, um zwei Begriffe zu benutzen, die dieser bei der Analyse der Rolle des Bundespräsidenten verwandt hatte und die Decker zitiert (S. 332).

Das Bessere ist der Feind des Guten - dieser Satz Voltaires ist der Ausgangspunkt für die folgenden Anregungen: Der Leitaufsatz über mehrheits- und konsensdemokratische Mechanismen bedarf der Erweiterung, damit die Frage „Konkordanz- oder/und Konkurrenzdemokratie?" in einem doppelten Sinne umfassend erörtert wird: zum einen deskriptiv, zum anderen präskriptiv. Wer die Rolle der Vetospieler systematisch entfaltet, stößt auf eine Vielzahl an Bremsmechanismen. Andere Aspekte werden wohl gestreift, jedoch nicht vertieft: Für einen vollständigen Blick zur „Architektur der deutschen Politik“ erscheint die Aufnahme eines Beitrages zur Rolle des grassierenden Rechtswegestaates sinnvoll. Gerade vor dem Hintergrund der tief in der deutschen Tradition verankerten Verrechtlichung der Politik ließe sich die „Architektur der deutschen Politik“ in ihrer Verschränktheit dadurch besser erfassen. Und der genius loci sollte einen Beitrag zur „Architektur der deutschen Politik“ vor dem Hintergrund der gewandelten Politischen Kultur und des Scheiterns der ersten deutschen Demokratie nahelegen. Der Bonner Gelehrte Karl Dietrich Bracher, dessen Arbeiten im Grenzgebiet von Politik- und Geschichtswissenschaft angesiedelt sind ${ }^{22}$,

21 Vgl. Theodor Eschenburg, Spielregeln der Politik. Beiträge und Kommentare zur Verfassung der Republik, Stuttgart 1987; siehe auch das nahezu vergessene, von Decker aber herangezogene Werk: Staat und Gesellschaft in Deutschland, Stuttgart 1960.

22 Vgl. Ulrike Quadbeck, Karl Dietrich Bracher und die Anfänge der Bonner Politikwissenschaft, Baden-Baden 2008. 
hatte Mitte der 1950er Jahre dazu ein wegweisendes Werk vorgelegt. ${ }^{23}$ Schließlich: Eine Analyse zur (voranschreitenden) Europäisierung der deutschen Politik ${ }^{24}$ gehört ebenso in einen solchen Band. Dann läge eine „geschlossene monographische Abhandlung" vor, die sich zur Erklärung vieler Verhaltensweisen im deutschen „Parteienbundesstaat" vollauf eignete. Bis dahin bleibt die Komposition unvollständig.

Auch wer stärker als Decker die Konkurrenzmechanismen als Problemlöser in den Vordergrund rücken möchte ${ }^{25}$, kommt nicht an der folgenden Erkenntnis vorbei: Diese Arbeit, die weder mit engagierten Stellungnahmen noch mit diskussionswürdigen Reformvorschlägen spart, zählt zu den wichtigsten problemorientierten „Regierungslehren“ in Deutschland. Sie liefert, wie gezeigt, vielfältige Anregungen. Der streitbare - nicht umstrittene - Demokratieforscher, der Politikwissenschaft betreibt, nicht Politik, wiewohl er vor pointierten Urteilen keineswegs zurückschreckt, steht mit seiner Strukturanalyse, die empirische und normative Sichtweisen souverän verbindet, in der ehrwürdigen „Bonner“ Tradition. ${ }^{26}$

23 Vgl. Karl Dietrich Bracher, Die Auflösung der Weimarer Republik. Eine Studie zum Problem des Machtverfalls in der Demokratie (1955), Düsseldorf 1984.

24 Vgl. Roland Sturm / Heinrich Pehle, Das neue deutsche Regierungssystem. Die Europäisierung von Institutionen, Entscheidungsprozessen und Politikfeldern in der Bundesrepublik Deutschland, Stuttgart 2006.

25 Vgl. dazu pointiert Thomas Darnstädt, Konsens ist Nonsens. Wie die Republik wieder regierbar wird, München 2006.

26 Vgl. Tilman Mayer / Volker Kronenberg (Hrsg.), Streitbar für die Demokratie. „Bonner Perspektiven" der politischen Wissenschaft und Zeitgeschichte 1959-2009, Bonn 2008.

\section{Innenleben des Bundestages: hellsichtige Nahaufnahmen, weiterführende Einsichten und wohlmeinende Elogen}

Schwarz, Hans-Peter (Hrsg.): Die Fraktion als Machtfaktor. CDU/CSU im Deutschen Bundestag 1949 bis heute, Pantheon Verlag, München 2009, 366 Seiten, € 16,95.

Schöne, Helmar: Alltag im Parlament. Parlamentskultur in Theorie und Empirie (Studien zum Parlamentarismus, Band 15), Nomos Verlagsgesellschaft, Baden-Baden 2010, 418 Seiten, $€ 59,-$.

Als arbeitsteilig strukturierte Organisationen gehören Parlamente zu den Eckpfeilern demokratischer Regierungssysteme. Zu den wichtigsten Akteuren im Parlament zählen die Fraktionen. Sie stellen nicht nur ein strukturgebendes Ordnungsprinzip dar, sondern sind zudem zentraler Garant zur Erfüllung der Parlamentsfunktionen. ${ }^{1}$ Für die Parlaments- und Regierungsmehrheit sind die sie tragenden Fraktionen das existenzsichernde Fundament; versagen sie der Regierung die Gefolgschaft, verliert die Exekutive ihre Handlungsfähigkeit. Als Instrument parlamentarischer Machtorganisation und Aufgabenzuordnung fungieren

1 Vgl. Suzanne S. Schüttemeyer, Fraktionen im Deutschen Bundestag. Empirische Befunde und theoretische Folgerungen, Opladen 1998. 